\title{
Educación e interculturalidad. Desafíos y retos hoy*
}

[Artículo de reflexión]

\author{
Raúl Fornet-Betancourt ${ }^{* *}$ \\ Reina Saldaña Duque $e^{* * *}$ \\ Ricardo Salas Astrain ${ }^{* * * *}$
}

Recibido: 01 de noviembre de 2020

Aceptado: 24 de febrero de 2021

Citar como: Fornet-Betancourt, R., Saldaña, R. y Salas, R. (2021). Educación e interculturalidad. Desafíos y retos hoy. Campos en Ciencias sociales, 9(1). https://doi.org/10.153332/25006681.6919

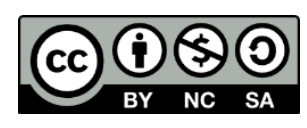

\section{Resumen}

Este texto es resultado del foro realizado por la Asociación Suramericana de Filosofía y Teología Interculturales (Asafti), el 10 de junio del 2020, "Polílogos Interculturales", cuya espiral, "Educación e Interculturalidad. Desafíos y retos hoy", invita a reflexionar en estos tiempos en los que la humanidad entera lucha por la vida, por el cuidado y por la supervivencia

\footnotetext{
* Este artículo nace del foro realizado por la Asociación Suramericana de Filosofía y Teología Interculturales (Asafti) el 10 de junio del 2020: "Polílogos Interculturales", en su espiral "Educación e Interculturalidad. Desafíos y retos hoy".

${ }^{* *} \mathrm{Ph}$. D. en Filosofía por las Universidades de Aachen y Salamanca; director del Instituto ISIS (Alemania); director de Concordia. Revista Internacional de Filosofía y coordinador del Programa de Diálogo Filosófico Norte-Sur. ORCID: https://orcid.org/0000-00015489-3199

*** Magíster de la Universidad de San Buenaventura, Cali, Colombia. Correos electrónicos: queen4419@hotmail.com, rsaldana@usbcali.edu.co; ORCID: https://orcid.org/0000-0002-6224-4911

${ }^{* * * *}$ Ph. D. en Filosofía de la Universidad de Lovaina y director del Doctorado en Estudios Interculturales de Universidad Católica de Temuco. Correo electrónico: rsalas@uct.cl; ORCID: https://orcid.org/0000-0003-4765-1567
} 
ante las desigualdades sociales, políticas, económicas y educativas de su contexto. Pensar la educación desde la interculturalidad posibilita preguntarse cómo conjugar las fuerzas desde los diferentes campos de pensamientos, prácticas y contextos. ¿Cómo centrar la mirada en el tejido social, en la historia, en la memoria de los pueblos, en las tradiciones y en los relatos de las comunidades para comprender que estas narrativas nos constituyen como sujetos y nos forman como ciudadanos críticos y habitantes del mundo? Es desde aquí que, para el caso de este documento, se provocan tres reflexiones: la relación pedagogía y educación desde una perspectiva intercultural; la educación y la interculturalidad desde los retos y desafíos contemporáneos a propósito del tránsito en el que vive la humanidad y el abordaje de lo educativo en clave de una relación intercultural desde la experiencia mapuche.

Palabras claves: pedagogía, interculturalidad, conocimientos, humanidad, colonización.

\section{Current Challenges in Education and Interculturality}

\section{Abstract}

This article is the result of the Intercultural Polylogue held by the South American Association of Intercultural Philosophy and Theology (ASAFTI), on June $10^{\text {th }}$, 2020, whose spiral: "Current challenges in Education and Interculturality", invites us to reflect on these times in which the whole humanity struggles for life, care, and survival in face of social, political, economic, and educational inequalities. To think about education from an intercultural perspective makes it possible to wonder how to combine forces from different fields of thought, practices, and contexts. How to focus on the social fabric, on the history and memory of the peoples, on the traditions, and the stories of the communities to understand that these narratives constitute us as subjects and form us as critical citizens and inhabitants of the world. From this starting point, three reflections are provoked: first, the relationship between pedagogy and education from an 
intercultural perspective; second, education and interculturality from the contemporary challenges humanity is undergoing; and third, the approach to education in terms of an intercultural relationship from the Mapuche experience.

Keywords: pedagogy, interculturality, knowledge, humanity, colonization.

\section{Introducción}

En este texto se discuten fundamentalmente dos cuestiones, esperando poder reflexionar sobre algunos tránsitos en los que el ser humano se encuentra actualmente. Por un lado, la educación - con sus grandes avatares, dinámicas, tendencias y direccionamientos que cuestionan el mismo campo de relaciones sociales y humanas - y, por el otro, la interculturalidad como posibilidad para seguir trabajando por el reconocimiento de los sujetos, sus culturas, sus saberes y sus contextos, indispensable en el campo educativo.

Estas dos cuestiones ponen en tensión a la escuela, el maestro, el estudiante y la comunidad y se preguntan: ¿de qué interculturalidad se habla en el contexto educativo?, ¿cómo generar espacios interculturales en el aula?, ¿qué es lo humano en la educación cuando se segrega, invisibiliza y niegan sus culturas?, ¿̇cómo pasar del discurso hegemónico en la escuela a prácticas plurales y diversas?, ¿cuáles son los currículos interculturales de los que habla hoy la educación?, porque, como indican Saldaña y Vélez (2019),

El reconocimiento de esta pluralidad en su dimensión de fuente de diversidad de saberes y conocimientos, de fuente de sentidos para la vida humana y para la comprensión de la convivencia social, así como para los modos de habitar la tierra, es para el pensamiento intercultural el punto de partida indispensable para convocar a fomentar una transformación de los procesos educativos. Transformación cuya finalidad sería precisamente la 
de posibilitar que los procesos educativos respondan realmente a situaciones contextuales y de este modo sean expresión y al mismo tiempo vehículos de la diversidad cultural de la humanidad. (p. 52)

Por consiguiente, la transformación educativa, desde la interculturalidad, supone el reconocimiento cultural y epistémico de las comunidades; propone nuevas prácticas y formas de conocimiento que miren los contextos, los territorios y los saberes y busca generar conocimientos en diálogos que partan de las realidades situadas de las colectividades.

\section{Algunas aproximaciones de la interculturalidad ${ }^{1}$}

En América Latina, existen - ante la diversidad cultural - procesos de reconocimiento desde la perspectiva política, gracias a los avances logrados en materia de derechos humanos y como resultado de las luchas sociales; sin embargo, esto esfuerzos, se sabe, han sido insuficientes ante la complejidad de lo social, en particular, de los procesos de mestizaje, la pérdida de la identidad cultural, la restitución de derechos, la creciente inequidad, la flexibilización del mundo laboral y la restricción de acceso a una educación mercantilizada, entre otros. Vale la pena puntualizar entonces que lo social no se restringe a lo cultural visto desde lo étnico. Ahora bien, desde los ochenta, la diversidad cultural con énfasis en lo étnico es un asunto de la mirada política, se propende por un reconocimiento legal que promueva las relaciones entre culturas y minimice su discriminación, exclusión e inferioridad en la sociedad denominada como mayor.

Es cierto que el reconocimiento jurídico es un comienzo, en el sentido en que por ejemplo países como Ecuador y en Bolivia han dejado de ser Estado

\footnotetext{
${ }^{1}$ Reflexiones que se amplían en la tesis Doctoral "Pedagogía e interculturalidad. Una lectura desde los procesos educativos en cuatro movimientos sociales", de 2020, del Doctorado en Educación de la Universidad de San Buenaventura de Cali, de la doctoranda Reina Saldaña Duque.
}

Campos en Ciencias Sociales

ISSN: 2339-3688 | e-ISSN: 2500-6681 | DOI: https://doi.org/10.15332/25394363

Vol. 9 N.o 1 | enero-junio de 2021 
nación, se han declarado naciones multiculturales y comunidades como la aimara o quechua han sido reconocidas en su identidad y se han aprobado programas de educación bilingüe, entre otras políticas. Sin embargo, a pesar de todo, esto resulta insuficiente ante la magnitud de la complejidad social, consecuencia de un largo proceso histórico de discriminación e invisibilización. En consecuencia, dichas políticas resultan poco efectivas por su incapacidad para atender las diferencias y necesidades de las comunidades, este panorama es esencial para la reflexión de la educación y con ello de la pedagógica en América Latina. De ahí que la interculturalidad desde la cual se reflexiona, no se queda en el reconocimiento, en la tolerancia o en la visibilización, sino que busca relacionar los sujetos, los mundos y las culturas, permitiendo ver la herencia y reclamando por culturas que piensen en el diálogo y encuentro con otras historias. La interculturalidad denuncia esas asimetrías y desigualdades y les apuesta a nuevas formas de reconceptualización, de refundar las estructuras sociales, epistémicas y de existencia, que ponen en escena modos culturales diversos de pensar, actuar y vivir en las comunidades, en los contextos. Por eso, la interculturalidad no es un hecho dado, está en permanente camino y construcción. Esta es la apuesta que se reclama en América Latina: una interculturalidad que establezca diálogos éticos, políticos, epistémicos y antropológicos en esta diferencia colonial y trabaje en la reconstrucción social y cultural de los pueblos y las comunidades.

Ahora bien, las aproximaciones que en tiempo presente se realizan sobre lo educativo y con ello sobre lo pedagógico suponen múltiples desafíos, dadas las reconfiguraciones que vive el mundo, como, por ejemplo, la confrontación entre las tendencias de la globalización del capitalismo neoliberal y la lucha por el reconocimiento de comunidades históricamente invisibilizadas. A partir de esta confrontación, hoy más que nunca emergen con fuerza las voces de las pluralidades largamente silenciadas. Por lo tanto, 
un acercamiento a lo educativo y pedagógico implica entrar en diálogo con la diversidad cultural y supone un cuestionamiento sobre la relación del ser humano con las culturas, los contextos y las epistemes, pues, al referirse la pedagogía a la educación, lo epistémico tiene un lugar central, ya que se trata de la configuración del ser humano desde sus capacidades de saber, pensar y conocer.

En clave a ello, la interculturalidad se aborda desde dos perspectivas: por un lado, la perspectiva antropológica y, por otro, la perspectiva epistémica. En la primera, se mira la relación entre el ser humano y la cultura, así como la relación entre la tradición y la historia; entonces, se parte por preguntar ¿cuál es la relación entre ser humano y la cultura?, donde se plantea como elemento a considerar la concepción de cultura, la cual presenta diversas nociones según la postura disciplinar que se adopte: cultura como arte, como literatura o como teoría. En este caso, es importante precisar que se apuesta por la cultura vida, vista desde Fornet-Betancourt (2012), y, en este sentido, se asume que la cultura se hace experiencia en las cotidianidades. Desde aquí, se denuncia la existencia de "una" cultura global hegemónica que mediante el sometimiento invisibiliza y niega la pluralidad cultural.

Las culturas son, pues, formaciones contextuales e históricas que tienen conocimiento de la contingencia de las respectivas tradiciones que las perfilan y que, en consecuencia, están en una relación histórico-relativa con lo que son... La cultura [...] tiene que ver con la gestación y el cultivo de las peculiaridades (formas de vida, sistemas de valor, etc.) en que se reconoce una determinada comunidad histórica. (Fornet-Betancourt, 2012, p. 81)

Es experiencia en tanto tiene en cuenta los vínculos, los entornos y las relaciones donde el ser humano se va formando y constituyendo como tal. Es desde esta grata experiencia de mundo donde se da entrada a la historia, el contexto, las prácticas de vida y la memoria de un pueblo. Entonces, el ser 
humano es un ser situado y un ser histórico que va configurando un vínculo a un espacio que lo contiene y lo delimita. De igual manera, es heredero de la tradición de su comunidad a partir de la cual ha tejido su autobiografía. Así, tanto el contexto como la historia se hacen vida en los procesos de socialización donde acontece la experiencia cultural, de ahí que se considere al humano un ser "situado" e "histórico". Aquí viene la segunda relación: la tradición y la historia. Esta se desarrolla en la medida en que el ser humano crece en su comunidad de referencia y en sus vivencias y experiencias culturales, justamente, por su participación en las tradiciones culturales que reconoce como propias. Frente a esta relación, es pertinente indicar que la tradición es entendida como acontecimiento histórico que vive en la memoria y se desarrolla a partir de las experiencias compartidas; en toda cultura hay una tradición "reconocida", que, para serlo, ha pasado por un proceso de confrontación y dominio sobre las "otras tradiciones" que han sido reprimidas, es decir, se da la lucha por la hegemonía y el poder de su transmisión.

Dado esto, el ser humano establece una relación dialéctica con su tradición que acontece entre el olvido y la resistencia. Cuando falla la memoria se da una fractura con la historia. Esta condición en el ser humano se hace presente cuando olvida sus raíces, las cuales son sustituidas, a través de un proceso de asimilación, por la unívoca visión de la cultura global. La apuesta entonces a nivel de la tradición es por la "destradicionalización" (FornetBetancourt), una destradicionalización que recoja visiones de la relación dada entre la tradición y la cultura; porque la relación no ha de ser ni de reducción, donde la cultura queda restringida a la tradición, ni de negación, invisibilizada en dicha cultura. La relación entre tradición y cultura estaría llamada a darse como un proceso de apertura y vitalidad, de ahí que "la labor de la destradicionalización busca mostrar que las culturas pueden y 
deben mantener una relación histórica, abierta y creativa, con sus tradiciones" (Fornet-Betancourt, 2012, p. 18).

Por otra parte, a nivel de la frontera, se propone la "fronterización" desde la resistencia en oposición a la "desfronterización" promovida por la globalización propia del capitalismo moderno. La fronterización es clave como lugar de resistencia que facilita la expresión de la pluralidad cultural. Se apuesta entonces por una resistencia activa de la memoria histórica, una en la que esta esté presente $y$, precisamente, no se abandone.

En la segunda, en la perspectiva epistémica, se centra la atención en la razón, el saber, el pensar y el diálogo, que se presentan desde un panorama general de la "travesía occidental hegemónica", desde donde la historia se ha narrado. El ahínco de la perspectiva epistémica se enmarca en dichas tradiciones; sin embargo, se dejan ver las realidades socioculturales y las voces de resistencia que dan apertura a nuevas apuestas en la contemporaneidad.

La razón se expresa como pretensión de pureza que para ser tal tiene que alejarse de lo experiencial y sensible. La consecuencia de este alejamiento, que es en realidad un encierro de la razón en su propio mundo categorial, es que el saber se afinca en la razón como verdad absoluta que deviene de la racionalidad y la lógica de la ciencia; se demarca la frontera entre la experiencia y la razón como insuperable vía de saber.

La apuesta que se hace desde América Latina y más precisamente desde los movimientos sociales es romper con los absolutismos. Esto implica hacer una crítica al abordaje de la cultura que condiciona y aniquila lo diverso, lo universal, lo particular y lo comunitario. Pero no es una crítica desde las márgenes de institucionalidad, si no desde cada sujeto, es decir desde la conciencia que estos tomen en relación a sus actos. 
En este mismo sentido, el pensar como acto de reflexión convoca a considerarnos no dentro del anclaje mecánico, lógico, utilitarista y civilizador occidental, sino desde un acto donde convergen sabidurías y saberes, en los que se recreen la autonomía, la concienciación, la libertad, la crítica y, sobre todo, una profundidad del pensamiento situado, que imagina y tiene un lenguaje "propio", aunque no sea reconocido.

Es necesario recordar que el pensamiento no es unívoco, lineal, uniforme ni dominante, al parecer se olvida que existen otras formas de pensamiento que no obedecen a las lógicas de la razón, pero que están allí en la multiplicidad de contextos. Se apuesta entonces por un diálogo que, si bien reconoce la historia y tiene memoria de la violencia epistemológica donde la razón, el saber y el pensamiento se apalancaron desde o-Occidente, propende hacia un reconocimiento de los saberes cuya matriz cultural es amplia e irriga las realidades, situaciones y experiencias en América Latina.

\section{La educación y la pedagogía desde una mirada intercultural}

La educación como escenario de tensiones de poderes se encuentra entre lo instrumental, la tecnologización y el desarrollo cientificista de la razón en pro del progreso de los mercados, condiciones que también afectan a América Latina, a partir de modelos pedagógicos cuyos currículos han sido dispositivos en los que se fortalecen estas condiciones: la ciencia, como mirada hacia el progreso, y la fe, incuestionable y dogmática.

Es importante reconocer entonces, en esta lógica educativa, cuáles han sido los lugares de la pedagogía, por lo que vale la pena reconocer las tendencias situadas en los diversos periodos históricos para determinar la externalización de eso que llamamos pedagogía, de ahí que se observen tres momentos destacados de la pedagogía en América Latina. Primero, el de las pedagogías impuestas en el periodo colonial, aquí se hace memoria del acto 
educativo no tan solo en la Colonia, sino un poco más atrás, donde la pedagogía se encuentra al servicio de la Iglesia y del Imperio español, periodo en el que se reflejan luchas económicas, políticas y de territorio, momentos que se amplían cuando se educa en la evangelización y cristiandad de los pueblos sometidos a cambiar sus costumbres, cultura, religión e, incluso, su lengua, sin mencionar la explotación y dominio de la tierra y el cuerpo de cada sujeto "incivilizado".

Segundo, las pedagogías aceptadas en el periodo republicano; en estas, se aboga por una nueva enseñanza, una que esté a favor de la modernidad, que permee la escuela y la institucionalice; ya el horizonte en el cual se encuentra la pedagogía deja de ser la fe, para posicionarse en el desarrollo, la racionalización e industrialización de un país cuyos modelos siguen siendo impuestos. Tercero, las pedagogías de la liberación desarrollada en la segunda mitad del siglo XX, en el contexto del desarrollo de la teoría_de la dependencia; donde la lucha, en cuanto a lo educativo, se centra en la resistencia a las políticas neoliberales impuestas y en el reconocimiento de las realidades de los sujetos, por tanto, vale la pena indicar cómo lo educativo, y con ello lo pedagógico, tiene propósitos políticos y sociales que pasan por el reconocimiento de la historia, el territorio y la fuerza en la autonomía de los sujetos.

En este sentido, se propone una lectura de las pedagogías desde las márgenes de la sociedad en movimiento, para lo cual se mencionan dos elementos. Uno, las pedagogías de las márgenes suceden en las orillas que trazan los procesos de exclusión social, cultural, política y económica, las orillas se definen de manera externa a aquellos confinados al "lugar de la exclusión"; y dos, históricamente en América Latina las formas de gobierno, de la mano de la educación, reproducen el orden social único, totalizante, reduccionista y excluyente de lo diverso. Se han llamado, a propósito de la tesis doctoral, pedagogías desde las márgenes en tanto se construye no 
desde los regímenes de Estados capitalistas, sino desde las bases de las sociedades en movimiento. Desde allí se piensa en la esencia del sujeto, los saberes y las prácticas situadas que han posibilitado un desprendimiento, un desheredar colonial. Estas otras pedagogías están a la base de una identidad, de una tradición que vincula las prácticas sociales e historias de los pueblos de América Latina.

Permitirse pensar en las pedagogías fuera de las márgenes de las disciplinas, las institucionalidades y los modelos en los que ha estado en América Latina supone una posibilidad de diálogo, aquí está el desafío y el reto, la posibilidad del diálogo desde otros lugares sociales diferentes a los del mundo moderno y colonial. Diálogo que convoca a reflexionar en torno al sujeto político desde su condición histórica, epistémica y situada, en contextos que se configuran en la lucha, la resistencia y la persistencia para la construcción desde estos otros modos de vida, de representación y de organización comunitaria y sociopolítica en los que han estado las pedagogías en América Latina.

Estas pedagogías desde las márgenes evidencian que existen en nuestra América pedagogías propias, es decir, pedagogías que se han dado a partir del giro antropológico y del giro epistémico. El primero, para trascender la postura capitalista, que ve al hombre como sujeto que consume, mas no dice ni crea pensamiento, sus experiencias y realidades no son susceptibles de generar epistemes. Y el segundo, que supone alterar los escenarios instituidos del conocimiento y dar apertura a las sabidurías emergentes. Estos giros se dan en la medida en que se establece una tensión entre la destradicionalización y la fronterización, lo que da lugar a dos elementos en el devenir de estas pedagogías; primero, las tensiones entre el lugar de la cultura política y la cultura vivida, que dan cuenta de la configuración de territorios que suponen la instalación de fronteras para la interlocución con 
los actores sociales que intervienen en el contexto educativo; segundo, el lugar de las epistemes totalizantes y las sabidurías emergentes.

Estos elementos se traducen en pedagogías desde las márgenes, las que a su vez se componen de una pluralidad de ritmos. Ritmos pedagógicos que se suscitan desde la destradicionalización: las experiencias, colectividades y afectividades. Y ritmos pedagógicos, que se suscitan desde la fronterización flexible, entendiendo la frontera como encuentro en la cual están ritmos como las voces y los diálogos.

\section{Educación e interculturalidad. Desafíos y retos hoy}

Antes de presentar en este apartado algunas breves reflexiones sobre la "Educación y la interculturalidad. Desafíos y retos hoy". Se hacen dos observaciones preliminares para nombrar los supuestos que están detrás de las reflexiones que se quieren compartir. El primero es un supuesto gnoseológico o de teoría del conocimiento porque tiene que ver con la calidad misma del conocimiento, como facultad humana que implica a todo el ser humano. Se habla de un presupuesto que tiene que ver con la calidad del conocimiento y se resume en la siguiente frase: "la calidad del conocimiento no depende de la cantidad de cosas que abarca", es decir, no depende de la magnitud extensiva que llegue a alcanzar, sino de la delicadeza de las experiencias desde las que participa y se implica el cognoscente en el curso total de la vida.

El segundo es un presupuesto ético, porque tiene que ver con la calidad de los maestros; o sea, con el carácter de las personas que asumen hoy en día esa tarea que se resume con el término de "educación". Este segundo presupuesto también se puede condensar en una frase: "instruir puede cualquiera, educar solo quien sea un evangelio vivo". Esta frase, que es de José de la Luz y Caballero, bien se puede considerar como la esencia de una ética de la educación, de ahí que se resume el presupuesto sobre la calidad 
humana de los maestros. Bien, se pasa entonces a unas breves consideraciones.

Lo primero que se debería hacer objeto de consideración es el tema mismo de este apartado: ¿qué significa eso de "desafíos" y "retos"? Para ello, la etimología de estas palabras da una pista importante, pues dice que son asuntos que convocan a una lucha, a un combate. Si es así, surge de inmediato la pregunta: ¿̇por qué ese "hoy", que evidentemente se refiere a esta época, desafía o reta a una lucha? Ante esta pregunta, la respuesta sería la siguiente: el hoy reta a los seres humanos porque es un tiempo que para el hombre genera, a su vez, contratiempos, el hoy es un tiempo de contratiempos. Lo que significa que ese hoy en el que se vive, se trabaja o se pretende educar es un tiempo en el que al mismo tiempo suceden percances y cosas malas, de modo que se siente el contratiempo de que "las cosas" no van por donde deberían ir, que lo que se suele llamar "curso del mundo" no va por el camino por el que los seres humanos quisieran que fuera. A luz de esta comprensión del "hoy", como un tiempo de contratiempo, se podría considerar la parte principal del tema: "educación e interculturalidad", para preguntarse si la educación y la interculturalidad son parte de los contratiempos que suceden en esta época o son por el contrario una salida, una manera de contrarrestar los contratiempos. También, aquí, se quiere arriesgar una respuesta, con el fin de contribuir a discusiones en común.

Por lo que toca a la educación, se diría, pues, que la educación es uno de esos contratiempos que desafían porque la educación por lo general está encerrada en marcos institucionales impuestos por la civilización hegemónica del capital. Por este enmarque, la educación es en gran parte un contratiempo que le sucede a muchas personas, en el sentido de que es una educación que instruye, sin educar. Se explica brevemente: la "educación" al servicio del sistema hegemónico instruye para que el ser humano 
funcione en el engranaje de la civilización hegemónica como ésta necesita que funcione para mantener su hegemonía.

En este contexto, se considera entonces que el reto fundamental que se le plantea a toda educación que pretenda ser liberadora es ser verdadera educación, en el sentido de formación humana, y no de mera instrucción o preparación profesional. ¿Cómo educar de manera que todos aprendan a salir del engranaje?, de la jaula donde han sido metidos, por decirlo de forma metafórica. En la línea de esta idea, la pedagogía de Freire, la pedagogía del oprimido y las pedagogías de las márgenes, en suma, las pedagogías liberadoras alternativas, tendrían que ser radicalizadas y prolongadas en la perspectiva de pedagogías del cautiverio, pedagogías para cautivos.

Es precisamente de cara al cumplimiento de esta tarea de radicalización de las pedagogías liberadoras que se conocen, donde, para los seres humanos, es necesario recordar el segundo supuesto del que se habló al principio. Pues, promover una educación liberadora radical y de manera consecuente como proceso de común liberación solo puede resultar de aquellos que en sí mismos son un evangelio vivo.

Se continúan las consideraciones con una anotación sobre la interculturalidad. Se empieza por hacer notar que la interculturalidad ha sido instrumentalizada por muchos modelos educativos hegemónicos, porque, al difundirse e imponerse el término en el espacio público mundial con el despertar de las múltiples reivindicaciones culturales de los pueblos, se pensó que la incorporación del término sería la mejor estrategia para dar la impresión de que se cumpliría con las demandas de justicia cultural. Bien, como reacción frente a esa instrumentalización por parte de organismos estatales y sus programas nacionales, por ejemplo, de "educación bilingüe", se acuño el término de interculturalidad crítica, para subrayar y dejar claro que se trataba y trata de una perspectiva nueva que se funda sobre bases 
alternativas de respeto a la pluralidad de todos los procesos educativos. Se insistía, pues, en la interculturalidad como un medio o una vía para hacer frente al contratiempo de la educación hegemónica. De donde se sigue que la interculturalidad debe ser entendida y practicada como un modo para educar, mostrando salidas del cautiverio, del estar encerrados, por ejemplo, en los horizontes de una lengua, de una cultura, de un color de piel o de una etnia, entre otros. Lo que a su vez quiere significar que la interculturalidad apunta a una manera de conocer que deja atrás las visiones cuantitativas del mundo. Para practicar la interculturalidad hay que romper con la lógica del más y menos, con la lógica de suma y resta, porque es una lógica cuantitativa, positivista y mecanicista. La interculturalidad rompe con los horizontes de esa lógica donde lo importante es medir y cuantificar, para proponer como "método" de vida y conocimiento la vía de la intensificación de las experiencias de vida humana. Se aclara que intensificar quiere decir, en ese "método" de la interculturalidad, energizar lo humano, aumentar o dejar que la energía humanizadora crezca.

Como última consideración, se indicaría finalmente que se debe ver en ese movimiento de crecimiento común en humanización la razón de ser última de un verdadero diálogo intercultural. Pues, si se dialoga a nivel de personas y de culturas, es porque se busca experimentar más integralidad e intensidad en las vidas y culturas. Dicho de otro modo: el diálogo intercultural presenta un camino para reencontrar la vida humana como la energía de convivencia que sostiene a los seres humanos.

Desde esta perspectiva, tratando de resumir lo expuesto en una frase, la interculturalidad subraya la necesidad de reorientar la educación en el sentido de un proceso abierto de formación humana convivencial, abriendo de este modo un nuevo horizonte para buscar una respuesta a los contratiempos de una educación hegemónica que hace a los seres humanos presos del sistema y coloniza las expectativas que pueden tener los pueblos. 


\section{Educación superior e interculturalidad crítica. Reflexiones desde el territorio interétnico Mapuche}

Para hablar de la educación y la interculturalidad, es preciso considerar algunos aspectos; es importante destacar los diálogos, debates y proyecciones que hacen parte de la experiencia de una forma de pensar la relación entre saber, pensamiento y mundo de vida. En este sentido, la filosofía intercultural ayuda a ver este proceso desde diferentes ópticas. Esto tiene que ver claramente con los diversos espacios y movimientos reflexivos que ha inspirado Raúl Fornet-Betancourt. A partir de él, y en deuda con otros como Rodolfo Kusch, Raimon Panikkar, León Olivé y Alcira Bonilla, Fidel Tubino y Joseph Estermann, se observa que se ha ido expandiendo en América un gran proceso de enraizamiento a los contextos, que son tan fundamentales en el ámbito educativo; en ese sentido, la filosofía y la teología intercultural representan un esfuerzo reflexivo y crítico por hacer una hermenéutica contextualizada de los territorios. Cuando se indica hermenéutica, se está haciendo referencia a un proceso de comprensión de otros espacios y tiempos que implican salir del terruño, y entender la idea de la travesía hacia otros mundos, ¿cómo los seres humanos efectivamente pueden atravesar las historias, lenguas y formas de ver el mundo para acercarse a otras culturas, lenguas, religiones y estéticas que a veces poseen raíces tan distintas? En la filosofía intercultural, esta experiencia del enraizamiento-desenraizamiento es un elemento crucial para que la filosofía intercultural ayude a despojar a los seres humanos de lo que les resulta familiar y cercano y, así, intentar hacer un viaje e ir al encuentro de lo que es familiar y cercano para los otros, esto presupone conectar con las raigambres de otros mundos.

En este sentido, se centra la mirada en los pueblos latinoamericanos que han pasado de ser territorios militarizados hace poco más de un siglo a ser ciudades modernas y globales que están rodeadas de muchas comunidades 
indígenas, afrodescendientes, populares y marrones, pueblos desconocidos e invisibilizados por el tenor modernista de la "urbe" y "la gran ciudad". ¿Cuántos mundos socioculturales coexisten en Lima, Rio de Janeiro, Caracas o México? Esto se ilustra en el caso del sur de Chile, en la ciudad de Temuco, donde a unos pocos kilómetros se encuentran territorios que pertenece o pertenecieron hasta hace poco a las comunidades mapuches. Es conocido que este territorio interétnico denominado por ellos Wallmapu está plagado de conflictos de diferentes tipos, causados por una larga historia de despojos, racismos y discriminación. El principal problema de la ciudad y de la región es lograr ver a una cultura diferente de la chilena hegemónica y pensar en las raigambres de un pueblo enraizado en la tierra. Eso significa ser mapuche en su lengua. Parte de los conflictos radica en que los proyectos sociales y políticos no presuponen una visión crítica del desarrollo, que siempre ha sido definido por los intereses hegemónicos, un desarrollo económico que hasta el día de hoy está vinculado a los sectores latifundistas, a las grandes empresas forestales de monocultivos y a los grandes proyectos hidráulicos y mineros, que no consideran los intereses de los propios habitantes originarios (Salas, 2017). ¿Es posible pensar una perspectiva de desarrollo de vida social y cultural donde sean consideradas las necesidades y expectativas que tiene en este caso el pueblo mapuche? Como en muchos territorios interétnicos, esto aparece en el discurso público y privado, pero no siempre se lo considera en serio, terminan siendo tan solo planes de las elites políticas de Santiago. Constatar esa asimetría es la gran preocupación, por sus consecuencias socioculturales, y es la utopía que mueve el accionar en el terreno formativo.

Ahora, se hace referencia a la experiencia intercultural más importante que la Universidad Católica de Temuco tiene entre sus estudios de posgrado. Se trata del Doctorado en Estudios Interculturales creado hace cinco años, donde veinte especialistas en ciencias sociales y humanas acompañan a 
dieciocho estudiantes profesionales que se están formando en esta perspectiva de hacer dialogar mundos y de apreciar el valor de las diferentes raíces culturales. Estos profesionales de todos los campos disciplinares están laborando en investigaciones interculturales que observan el encuentro-desencuentro desde una mirada reflexiva y crítica. Los estudios interculturales ayudan a pensar en un gran proceso de reflexión inter y transdisciplinaria que permita ver las grandes posibilidades que tiene el Wallmapu, para que se transite y dialogue entre mundos distintos. Hasta ahora dicha travesía ha sido llevada adelante por personas y comunidades mapuches, pero no se ha logrado ese mismo viaje por parte de la sociedad chilena. Esta asimetría de los viajes interculturales también tiene dificultades y escollos. Todo eso tiene que estar presente en el diseño formativo.

¿Cómo se dialoga con un pueblo indígena que tiene una historia distinta a la nuestra, que tiene una lengua completamente distinta al castellano y que tiene otros conocimientos que son definitivamente diferentes a los nuestros, que, como cultura dominante, vive y se nutre del pensamiento occidental? Solo para poner un ejemplo, muchos estudiosos de la cultura indígena ponen en duda que los pueblos hayan logrado un desarrollo de sus potencialidades reflexivas y críticas, si uno mira por ejemplo el pueblo mapuche y considera la originalidad de lengua, se observa que hay dos expresiones que son muy significativas y desconocidas para nosotros (Salas, 2009). Ellos tienen la expresión rakizuam, que significa la colección de todos los conocimientos que los seres humanos pueden alcanzar, pero agregan una expresión muy distinta que es la noción kimün, que significa un saber en su profundidad, en sus relaciones más éticas y sapienciales. Desde esta palabra se construye el concepto kimche (persona sabia) que tiene una sintonía y sinergia con la espiritualidad que está presente en el conjunto de la humanidad. 
Si se consideran estas potencialidades semánticas, se ve entonces que en el pueblo mapuche existe una serie de posibilidades de poder dialogar con las nociones de ciencia, de conocimiento y de filosofía. Para mostrar que esto es posible en un espacio universitario, se propone analizar la experiencia de un curso que se lleva a cabo para los estudiantes de cualquier carrera de pregrado de la universidad. Se trataba de proponer un curso que tendría como objetivo buscar un diálogo entre la filosofía europea y la filosofía mapuche. Se conversó con una autoridad espiritual para ponerse de acuerdo, pero faltaba poner un título al curso. Se había propuesto "Filosofía Occidental-Filosofía Mapuche”, pensando entonces que al poner ambas nociones juntas estaba resuelto el asunto (Salas y Marielo, 2011). Pero al contactar a la autoridad mapuche, quien es un sabio mapuche, él dice "¿cuál va a ser la óptica de tal relación?” y agrega “cexisten las condiciones para poder dialogar?”. Esta historia se comenta casi siempre para indicar lo complejo que resulta dialogar de veras, lo que parece bastante potente, frente a una concepción simplista del diálogo interétnico e intercultural. A veces se piensa que solo basta estar en el mismo salón de clases para que haya diálogo, pero realmente las preguntas son: cexisten las condiciones?, ¿este es el comienzo del diálogo?, ¿̇se está de veras mirando a los otros o a nuestros imaginarios o espejismos? Estas son las problemáticas que requieren mucha mayor reflexión y que se dan en este tipo de experiencias de encuentros culturales.

En el terreno de un posgrado, esto alcanza otras dificultades más complejas de tipo metodológico y epistemológico, respecto a cómo se avanza en el diálogo y en el conocimiento recíproco. La pregunta que va apareciendo en las investigaciones de los estudiantes es ċcuál es el tipo de metodología intercultural para alcanzar determinados resultados al que estamos obligados por ser un doctorado universitario? El asunto no es sencillo, porque cada vez que se pregunta a los estudiantes sobre la metodología de 
investigación, ellos retornan a las metodologías occidentales no por un camino que recoja plenamente la voz de actores y los tipos de conocimientos que los sujetos y comunidades tienen. La gran cuestión no es solo levantar temáticas y contenidos, sino mirar cómo se es crítico de una preocupación epistemológica y metodológica, para levantar los saberes invisibilizados y las prácticas culturales de contacto que tienen las propias comunidades, en este sentido, ir desaprendiendo de aquellas formas metodológicas y epistemológicas de hacer investigación científica. Pero, los programas de posgrado sometidos al control de un paradigma de la ciencia mundial aparecen como menos científicos. Para la acreditación de una Institución de Educación Superior(IES) es preciso explicar muchas veces lo que implica una investigación intercultural, ya que criterios de las prácticas de investigación siguen siendo todavía monoculturales.

Para finalizar esta relación entre educación e interculturalidad como parte de esta experiencia en Wallmapu, es preciso indicar que lo más importante del conocimiento para el mundo mapuche se podría enunciar sintéticamente, no es la cantidad de conocimientos lo que define su profundidad, sino la intensidad de dicho saber para hacer al hombre mejor persona (che), esto es lo esencial. En este marco de diálogo dialogante, como gustaba decir Panikkar, es la calidad de la relación que yo tengo con alguien diferente. En este sentido, cuando un mapuche habla en su lengua y habla del mapun kimün, lo que indica es que conocer involucra varias dimensiones de sus propias raigambres, donde, por un lado, se necesita saber quién es el otro que me habla y me puede enseñar y, por otro, quién soy yo, que puedo hablar, enseñar y ayudar eventualmente a ese otro. Por eso, la humanidad está en unos intersticios donde en el diálogo cada uno enfrenta su propia experiencia local. Ahora, mientras no haya esa travesía verdadera y no haya una transformación de las miradas, no se podrá avanzar en investigaciones genuinas que permitan comprender los saberes y 
las prácticas de las otras culturas y la humanidad quedará atrapada en un currículo occidental y monocultural.

\section{Consideraciones}

Con el ánimo no de concluir, sino de generar reflexiones para próximas conversaciones, se parte por poner en cuestión tres asuntos para seguir conversando. Uno, que la educación en clave a procesos interculturales posibilita dar sentido a las voces, las culturas, los sujetos, las prácticas y las situaciones que se dan en los contextos, en las comunidades; de ahí que,

[...] pensar la interculturalidad en el campo de la educación en América Latina, es darle sentido a la construcción de las convivencias entre las culturas que subyacen en cada territorio, en cada práctica, en cada relación entre sujetos de una o más comunidades. (Saldaña y Vélez, 2019, p. 55)

Dos, la interculturalidad centra la educación en un escenario de tensiones, relaciones y expresiones desde donde se puede transformar el tejido social. De ahí que valga la pena preguntarse ¿cómo la educación, y con ello la escuela, afecta a las relaciones, las subjetividades, las diversidades de los contextos, los territorios y las comunidades?, porque

La figura escuela y la educación se alejan de los límites del adentro y el afuera, de lo local y lo global, de lo propio y lo extraño, de la identidad y la alteridad, de lo sólido y lo líquido, de lo lento y lo veloz. La complejidad de ellas son los cruces, clivajes y mixturas espaciotemporales que están redefiniendo el campo de juego de lo educativo. (Saldaña, Rubio, Quintero y Rocha de Brito-Rodrigues, 2020, p. 9)

Y tres, se apuesta por una educación intercultural como posibilidad de recuperar las experiencias y las culturas, como espacios desde donde se resignifica la memoria, las tradiciones y las culturas mismas, desde donde se 
apuesta por una formación crítica y política de los pueblos y las comunidades.

\section{Referencias}

Fornet-Betancourt, R. (2012). Interculturalidad, crítica y liberación. Editorial Aachen Wiss-Verl.

Salas, R. (2009). Pensamiento mapuche. En C. Bohorquez, E. Dussel y E. Mendieta (Eds.), Historia del pensamiento filosófico latinoamericano, del Caribe y "latino" (130o200o) (pp. 41-46). Crefal; Siglo XXI Editores.

Salas, R. y Marileo, A. (2011). Filosofía occidental y filosofía mapuche: iniciando un diálogo. Revista Isees, (9), 119-128.

https://dialnet.unirioja.es/servlet/articulo? codigo $=3777538$

Saldaña, R. y Vélez, C. (2019). Educación e interculturalidad. En J. M. Hernández Díaz, A. Pozzer y E. Cecchetti (Coords.), Migración, interculturalidad y educación: impactos y desafios (pp. 41-58). Ediciones Universidad de Salamanca.

Saldaña, R., Rubio, J. C., Quintero, M. L. y Rocha de Brito-Rodrigues, L. (2020). Clivajes educativos: expansión, silencios y sujetos políticos. Praxis, 16(2), 215.225.

https://doi.org/10.21676/23897856.3262

Campos en Ciencias Sociales

ISSN: 2339-3688 | e-ISSN: 2500-6681 | DOI: https://doi.org/10.15332/25394363

Vol. 9 N. ${ }^{\circ} 1$ | enero-junio de 2021 